\title{
S-wave envelope broadening characteristics of microearthquakes in the Canary Islands
}

\author{
Arantza Ugalde \\ Institute of Earth Sciences “Jaume Almera” - CSIC. Lluís Solé i Sabarís, s/n, \\ 08028 Barcelona, Spain \\ Tel. +34 934095410 \\ Fax. +34934110012 \\ E-mail: augalde@ictja.csic.es
}

\begin{abstract}
This study analyzes the S-wave envelope broadening characteristics of 290 earthquakes recorded by 14 stations of the Spanish National Seismograph Network in the Canary Islands region. The S-wave peak delay time $\left(t_{p}\right)$ and envelope duration $\left(t_{q}\right)$ parameters are evaluated phenomenologically to infer the strength of velocity inhomogeneities of the medium along each seismic ray path. Crustal ( $18 \mathrm{~km}$ ) and upper mantle (18 $80 \mathrm{~km})$ events are analyzed separately. Results in the frequency range 1 to $12 \mathrm{~Hz}$ for hypocentral distances from 30 to $600 \mathrm{~km}$ show that both $t_{p}$ and $t_{q}$ increase according to a power of hypocentral distance and they are independent of frequency. The spatial distribution of the peak delay time reveals weak strength of heterogeneity in most of the region at shallow depths. Relatively strong inhomogeneous zones are generated under the island of Tenerife and Gran Canaria at depths of 11-22 km.
\end{abstract}

Keywords: coda waves - scattering - envelope broadening - Canary Islands

\section{Introduction}

Some observed characteristics of high frequency $(>1 \mathrm{~Hz})$ seismograms from local and regional earthquakes can be explained by the presence of heterogeneities in the earth's lithosphere with wide characteristic scales compared with the seismic waves wavelength. The scattering process at small-scale random heterogeneities of the lithosphere manifests in the existence of coda waves, which have proved to be useful for the quantitative characterization of the spatial heterogeneity in the lithosphere. Since the pioneering works of Aki (1969) a number of models have been developed to determine the spectral structure of the random heterogeneity in the earth (see Sato and Fehler 1998 for a detailed review). The radiative transfer theory, which describes the energy transport through a random heterogeneous medium neglecting phase information, provides an adequate framework for the modelling of high-frequency seismogram envelopes (Wu 1985). On this basis, 
numerous studies have been performed to separate the scattering due to random heterogeneities and the energy loss due to intrinsic absorption in several regions of the world (e.g. Ugalde et al. 2007 and references therein). Most of these practical approaches assume isotropic scattering and uniform distribution of scatterers and intrinsic absorption. Scattering from randomly and non-uniformly distributed heterogeneities has also been studied to characterize structures of scattering loss (e.g., Nishigami 1991; Ugalde et al. 2006) although a uniform distribution of intrinsic absorption is also assumed.

Another evidence for the existence of heterogeneities in the lithosphere is the observed increase of the duration of the incoherent direct wave group with travel distance. This envelope broadening phenomena is produced by multiple forward scattering and diffraction that are expected to be predominant on the wave propagation through a random medium whose correlation distance is longer than the seismic wavelength (Obara and Sato 1995). After Sato (1989) two parameters are used to quantify the non-uniform scattering structure of the earth by the analysis of envelope broadening: the peak delay time, $t_{p}$, and the envelope duration, $t_{q}$. The peak delay time is the time lag from the $S$-wave onset to the maximum amplitude arrival, and it is known to be a good measure of scattering strength (e.g., Gusev and Abubakirov 1999a,b; Takahashi et al. 2007). The envelope duration is defined as the time difference between the S-wave onset and when the envelope decreases to half of its maximum amplitude. This parameter also reflects the scattering strength of the medium although it is greatly affected by the intrinsic absorption (Obara and Sato 1995, Saito et al. 2002). Envelope broadening characteristics are well modeled by applying the Markov approximation to the parabolic wave equation (Sato 1989; Fehler et al. 2000; Saito et al. 2005). A method based on the radiative transfer theory has also been developed to simulate the envelope broadening caused by forward scattering and to reconstruct the distribution of the scattering properties of the medium (Gusev and Abubakirov 1999a,b; Petukhin and Gusev 2003). Several observations on Swave envelope broadening have been reported: Obara and Sato (1995) analyzed the peak delay and duration times in the Kanto-Tokai region of Japan, and found that the envelope broadening in the backarc side of the volcanic front is larger than in the forearc side; Takahashi et al. (2007) found a path dependence of the Swave peak delay times in relation to the distribution of Quaternary volcanoes in 
northeastern Japan; and Tripathi et al. (2010) characterized the S-wave broadening effect based on the signal duration (e.g., Petukhin and Gusev 2003) in northeastern Japan.

In this work we perform a phenomenological study on the envelope broadening characteristics of microearthquakes in the Canary Islands archipelago. The scattering characteristics in this region were previously studied by Canas et al. (1998), who estimated the intrinsic absorption and scattering loss by means of the Multiple Lapse Time Window method (Hoshiba et al. 1991) within the framework of a model of multiple isotropic scattering in a medium with uniform distribution of scattering and absorption properties. They found that the intrinsic absorption is the dominant attenuation effect for all the studied frequencies in the region. In the following we examine the S-wave peak delay and duration times of microearthquakes in relation to the spatial distribution of ray paths and we discuss the results with previous findings in the Canary Islands region.

\section{Geotectonic setting and data}

The Canary Islands are an intraplate volcanic archipelago located between latitudes $27^{\circ} 38^{\prime} \mathrm{N}$ and $29^{\circ} 25^{\prime} \mathrm{N}$ and longitudes $13^{\circ} 20^{\prime} \mathrm{W}$ and $18^{\circ} 9^{\prime} \mathrm{W}$. The Canary archipelago comprises seven main islands and several islets that form a chain extending for about $500 \mathrm{~km}$ across the eastern Atlantic, with its eastern part located close to the western continental African edge (Figure 1). The islands lay on Jurassic age oceanic lithosphere, between the isochrones S1 ( 170-175 Ma, Roest et al. 1992) and M25 ( 156 Ma, Verhoef et al. 1991) and were formed during the Neogene through multiple volcanic episodes. All but one of the seven Canary Islands have been volcanically active in the last million years and four of them show a record of eruptions in the last five centuries.

Due to its complexity, the geodynamic setting of the Canary archipelago is not yet well understood, being still a controversial matter of debate. Historically, four hypotheses have been proposed to explain the magmatic activity of the Canary Islands: (1) the mantle plume model (Morgan 1971; Burke and Wilson 1972), (2) the propagating fracture hypothesis (Anguita and Hernán 1975), (3) the uplift tectonic model (Araña and Ortiz 1986), and (4) the local extensional ridge theory (Fúster 1975). Although the hot spot is the most popular model among 
scientist, every hypothesis, including the mantle plume model variants (Hoernle and Schmincke 1993) and the unifying model (Anguita and Hernán 2000), suffers from inconsistencies with the local and regional geology. But whatever the case, at present it is widely believed that regional tectonics may have played a role in the evolution of the Canary Islands (Anguita and Hernán 2000).

Seismic activity in the Canary Islands is moderate with earthquake magnitudes usually $<5$. Volcanic and tectonic events occur in the region, being the fault between the islands of Tenerife and Gran Canaria the source of most of the tectonic seismic activity in the region since 1989. An important NE-SW fracture has been traditionally interpreted in the channel between Tenerife and Gran Canaria (Bosshard and McFarlane 1970; Mézcua et al. 1992). An earthquake of magnitude $5.2\left(M_{\mathrm{b}} \mathrm{Lg}\right)$ occurred in this zone on 9 May 1989 which was followed by a large number of aftershocks. Mézcua et al. (1992) obtained a reverse strikeslip focal mechanism of the main shock corresponding to a NE-SW fault. The solution differs significantly from the focal mechanism given by the CMT catalogue (Dziewonski et al. 1990), which implies the presence of an E-W or a NW-SE fault. However, Mézcua et al. (1992) solution fits well the distribution of aftershocks and the regional seismicity trend.

In recent decades, together with local geophysical surveys, many regional marine geophysical studies have been conducted in the archipelago using seismic, gravity and magnetic data (e.g., Dañobeitia et al. 1994; Ranero et al. 1995; Socías and Mézcua 1996; Canales and Dañobeitia 1998; Carbó et al. 2003). Canas et al. (1995) and Canas et al. (1998) first analyzed short-period analogical seismic recordings from tectonic earthquakes in the archipelago to estimate $\mathrm{S}$-wave attenuation in the region using coda waves. For this study, 290 tectonic earthquakes that occurred in the period 2000-2006 in the Canary archipelago were selected. The vertical-component, digital waveforms used were recorded by 14 short-period and broadband stations of the National Seismic Network that belongs to the Spanish Instituto Geográfico Nacional (IGN). Earthquakes with $m_{b}$ magnitudes ranging between 2.0 and 4.1 and having hypocentral distances less than about $600 \mathrm{~km}$ and focal depths less than $80 \mathrm{~km}$ were analyzed. Table 1 shows the velocity model used by the IGN for earthquake locations in the Canary Islands region. Epicenters, stations and ray paths used in this study are shown in Figure 1. Figure 2 plots an example of one analyzed seismogram. 


\section{Data analysis and results}

\subsection{Peak delay time and envelope duration measurement}

In order to better understand possible small variations of the S-wave peak delay time and envelope duration parameters with frequency, we chose several narrow frequency bands for the analysis. The number and extension of the frequency bands used are limited by the short-period instrument characteristics available. In this study, each seismogram was bandpass-filtered over the four frequency bands:

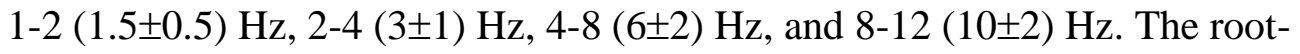
mean-squared (rms) seismogram envelopes were computed by applying a $0.1 \mathrm{~s}$ spaced moving time window of width twice the centre period of each frequency band. Then, the time lag in seconds from the S-wave onset to the maximum amplitude arrival $\left(t_{p}\right)$ and the time when the rms envelope decays to the half of the peak value $\left(t_{q}\right)$ were measured. We used the S-wave arrival time picked routinely at the IGN. To automate the process, we measured $t_{p}$ in a $30 \mathrm{~s}$ time window starting from the $\mathrm{S}$-wave onset. The algorithm also searched for $t_{q}$ as the maximum time from which the envelope is below the half of the maximum rms amplitude. The rms amplitudes for a noise window of $10 \mathrm{~s}$ before the P-wave arrival were also computed for each frequency band. Envelope data with amplitudes less than twice the signal to noise ratio were discarded. After this quality control procedure only 561 good quality seismograms were finally kept. The paths used are plotted in Figure 1 and Figure 2 shows an example of the processing for measuring $t_{p}$ and $t_{q}$.

\subsection{Hypocentral distance dependence of the envelope broadening parameters}

Figure 3 plots the variation of $t_{p}$ and $t_{q}$ as a function of hypocentral distance, $r$, in logarithmic scale, for each studied frequency band and for two hypocentral depth ranges: crustal ( $18 \mathrm{~km})$ and upper mantle (18 $80 \mathrm{~km})$ events.

Figure 3 also shows the least-squares fitting lines for $t_{p}$ and $t_{q}$ against hypocentral distance using the equation 
Data show a linear trend of $t_{p}$ and $t_{q}$ as commonly observed in logarithmic scale plots for other regions (e.g., Saito et al. 2002; Takahashi et al. 2007). This means that, in spite of the scatter of the data, both $t_{p}$ and $t_{q}$ increase according to a power of hypocentral distance for all the studied frequency bands and focal depth ranges. Table 2 shows the estimated regression coefficients and along with their error estimates. The correlation coefficients of the fit are small, especially for the 1-2 Hz frequency band in the case of crustal events. Figure 4 shows a comparative plot of the estimated $B_{p, q}$ coefficients as a function of frequency. It is noticed that the mean values for $B_{p}$ and $B_{q}$ are greater for upper mantle events than for crustal ones but, taking into account the error bounds, no significant differences are observed for all the analyzed frequencies except for the 1-2 $\mathrm{Hz}$ frequency band. Table 2 shows that, in general, the mean values for the regression slopes $B_{p}$ are greater than the mean $B_{q}$ for the studied frequency range, thus indicating that the dependence on the hypocentral distance is slightly stronger for the peak delay time than for the envelope duration parameter except for the 8$12 \mathrm{~Hz}$ frequency band in the case of upper mantle events. The difference, however, is not significant when taking into account the error intervals of the estimation.

Figure 5 shows examples of seismic envelopes observed on different ray paths for the frequency band 2-4 Hz. It is noticed that for event numbered (1) (hypocentral depth $\sim 20 \mathrm{~km}$ ) the peak delay time, $t_{p}$, is greater on the ray path to station CCAN $(r=50 \mathrm{~km})$ than on the ray path to station EBAJ $(r=55 \mathrm{~km})$, which is located at the north of Tenerife island. As for event numbered (2) (hypocentral depth $~ 30 \mathrm{~km}$ ), the peak delay time is smaller on the ray path to station EOSO $(r=75 \mathrm{~km})$, than on the ray path to station CICO $(r=77 \mathrm{~km})$, that propagates beneath Teide volcano in Tenerife. Path dependence is not observed for the envelope broadening parameter, $t_{q}$, in these examples.

\subsection{Frequency dependence of the S-wave envelope broadening}

In order to study the dependence on frequency of $t_{p}$ and $t_{q}$, it is necessary to remove the contribution of the hypocentral distance on each measurement. Following Obara and Sato (1995) one approach is to normalize the observed parameters by their values in a reference frequency band. Figure 6 plots examples of the normalized $t_{p}$ and $t_{q}$ parameters for six seismic stations distributed 
throughout the archipelago, where the 4-8 $\mathrm{Hz}$ frequency band has been chosen as the reference. Solid circles and open triangles plot the individual measurements and average values of $t_{p}$ and $t_{q}$, respectively, for earthquakes with focal depths greater than $18 \mathrm{~km}$. The scattering of the normalized measurements is large, which may be related to the location of the earthquake. It is observed that the broadening of seismogram envelopes shows no clear frequency dependence at any station in the Canary Islands region.

\subsection{Spatial distribution of peak delay time}

In order to determine the locations of strong inhomogeneity producing large $t_{p}$ values, it is necessary to quantitatively characterize the path dependence of each peak delay time parameter. Let be the $j$ th observed value of the peak delay time. Following Takahashi et al. (2007) it is possible to calculate the logarithmic deviation of $t_{p}$ as

Where and are the regression coefficients of equation (1). in equation (2) represents the relative accumulated scattering contribution along each ray path $j$.

Let's divide the medium into many small blocks. By considering all the ray paths available, one block will be passed through by several ray paths that correspond to different values of . Following Takahashi et al. (2007) the smallest value will characterize the strength of heterogeneity of each block, because the delayed peak arrival time cannot be further shortened by other weak heterogeneities along the ray path.

In this study, we constructed the grid by dividing the region into $N=60 \times 30 \times 7$ cells of dimensions $0.1^{\circ} \times 0.1^{\circ}$ in horizontal and $11 \mathrm{~km}$ in depth. Then, we calculated the ray paths by assuming straight trajectories and we measured the minimum value for each block. Only the cells crossed by more than 3 ray paths were kept. Figure 7 shows the minimum value distribution of using upper mantle events for the frequency bands $2-4 \mathrm{~Hz}, 4-8 \mathrm{~Hz}$, and $8-12 \mathrm{~Hz}$ at depths of 0-11 km and 11-22 km. Regionalization at $1.5 \mathrm{~Hz}$ center frequency is not shown due to the few data available. Blue colors represent the 
blocks with small values of , whereas orange colors indicate regions

of large $\quad$ values. Figure 7 shows that the strength of heterogeneity,

characterized by , is weak in most of the study region. These results will be discussed in the next section.

\section{Discussion}

Envelope broadening has been parameterized by the time lags of the peak arrival and the half maximum amplitude arrival from the S wave onset. Seismogram envelopes from tectonic earthquakes in the Canary Islands archipelago have been analyzed to establish the hypocentral distance and frequency dependence of the time lags measurements. Crustal ( $18 \mathrm{~km})$ and upper mantle (18 $\mathrm{km}$ ) events have been analyzed separately for four frequency bands from 1 to 12 $\mathrm{Hz}$. Results show that both $t_{p}$ and $t_{q}$ increase according to a power of hypocentral distance in the region for the distance range $30 \mathrm{~km}$ to $600 \mathrm{~km}$. Taking into account the error intervals, no significant differences in the rate of increase are found between the two focal depth categories considered. Figure 8 shows a comparison of the slopes of the regression lines of obtained in this work for upper mantle events and in other studies. It can be observed that the coefficients are smaller than those obtained by Saito et al. $(2002,2005)$ in the northeastern Honshu region of Japan and by Takahashi et al. (2007) beneath Quaternary volcanoes in northeastern Japan, but they are inside the bounds obtained by Obara and Sato (1995) in the Kanto-Tokai region of Japan for some stations except for the 2-4 Hz frequency band. The focal depth of the earthquakes analyzed in this study, however, is shallower than those used in Japan by the authors mentioned above.

As suggested by Sato (1989) seismogram envelopes broaden with increasing frequency in the case of media characterized by non-Gaussian autocorrelation functions (ACF). Therefore, the envelope broadening frequency dependence may be related to the spectral properties of the random inhomogeneous velocity structure. The envelope broadening parameters, $t_{p}$ and $t_{q}$, obtained in this study do not show frequency dependence for any of the stations considered in the study area. This result may indicate that random inhomogeneity in the Canary Islands region is represented by a Gaussian ACF. An analogous 
conclusion was reached by Canas et al. (1998) who separately estimated the contribution of intrinsic absorption and scattering to total attenuation using an independent data set in the same region. They found that the decrease of scattering attenuation was faster than with increasing frequencies, which implies that the medium may be characterized by a Gaussian ACF (Wu, 1985).

Path dependence of S-wave envelopes has been investigated by calculating the 3D distributions of the peak delay time in the region. The relative strength of accumulated scattering contribution along each ray path has been characterized by the minimum value distribution of the logarithmic deviation . Results plotted in Figure 7 show that most of the region presents small values of , which correspond to weak inhomogeneities for all the studied frequency bands. Some relatively large values distributed throughout the study region are found at depths of 0-11 km for all frequencies. Closely looking at the spatial changes at depths of 11-22 km, the regions of stronger are concentrated under the island of Tenerife and Gran Canaria at the 2-4 Hz band and under Tenerife at the 4-8 $\mathrm{Hz}$ frequency band. is also strong near Fuerteventura at $3 \mathrm{~Hz}$ center frequency for shallow depths. The diffraction phenomena are related to velocity inhomogeneities having a scale larger than the seismic wavelength (e.g. Takahashi et al. 2007). This means that, for the frequency range analyzed in this study and if we consider an average S-wave velocity of $4 \mathrm{~km} / \mathrm{s}$ (e.g. Canas et al. 1998) strong heterogeneities with a scale from several hundreds of meters to a few kilometers may be present in the region. These results are in agreement with Canas et al. (1998) who found a low scattering attenuation in the Canary Islands and related the strong frequency dependence of the attenuation parameters obtained to the size of the heterogeneities in the region, which would be at least comparable to the wavelength of the lowest analyzed frequencies, or about $2.6 \mathrm{~km}$.

\section{Conclusions}

We have measured the peak delay time and envelope duration parameters using Swave envelopes from tectonic earthquakes recorded at the Canary Islands archipelago. These parameters are found to increase with hypocentral distance and they are independent of frequency. These observations may indicate that the medium is characterized by a Gaussian autocorrelation function. A regionalization 
procedure of the peak delay time shows weak random velocity inhomogeneities throughout most of the study region. Some heterogeneous zones distributed throughout the archipelago are revealed at depths of $0-11 \mathrm{~km}$ and under the island of Tenerife and Gran Canaria at depths of 11-22 km.

\section{Acknowledgements}

We are very grateful to Carmen López and the people of the Instituto Geográfico Nacional, Spain, for their support during the data selection process. Funds provided by REPSOL are supporting A. Ugalde. Two anonymous reviewers made constructive comments that helped to improve the paper. 


\section{References}

Aki K (1969) Analysis of the seismic coda of local earthquakes as scattered waves. J Geophys Res 74:615-631.

Anguita F, Hernán F (1975) A propagating fracture model versus a hot-spot origin for the Canary Islands. Earth Planet Sci Lett 27: 11-19.

Anguita F, Hernán F (2000) The Canary Islands origin: a unifying model. J Volcanol Geotherm Res 103: 1-26.

Araña V, Ortiz R (1986) Marco geodinámico del volcanismo canario. An Física Vol Esp 82: 202231.

Bosshard E, MacFarlane D J (1970) Crustal structure of the western Canary Islands from seismic refraction and gravity data. J Geophys Res 75: 4901-4918.

Burke K, Wilson JT (19729 Is the African plate stationary? Nature 239: 387-390.

Canales JP, Dañobeitia JJ (1998) The Canary Islands swell: A coherence analysis of bathymetry and gravity. Geophys J Int 132: 479-488.

Canas J A, Pujades LG, Blanco MJ, Soler V, Carracedo JC (1995) Coda Q distribution in the Canary Islands. Tectonophysics 246: 245-261.

Canas J A, Ugalde A, Pujades LG, Carracedo JC, Soler V, Blanco MJ (1998) Intrinsic and scattering seismic wave attenuation in the Canary Islands. J Geophys Res 103: 15037-15050.

Carbó A, Muñoz-Martín A, Llanes P, Álvarez J, EEZ Working Group (2003) Gravity analysis offshore the Canary Islands from a systematic survey.Mar Geophys Res 24: 113-127.

Dañobeitia J J, Canales JP, Dehghani GA (1994) An estimation of the elastic thickness of the lithosphere in the Canary Archipelago under admittance function. Geophys Res Lett 21: 26492652.

Dziewonski AM, Ekström G, Woodhouse JH, Zwart G (1990) Centroid-moment tensor solutions for April-June 1989. Phys Earth Planet In. 60: 243-253.

Fehler M, Sato H, Huang LJ (2000) Envelope broadening of outgoing waves in 2D random media: a comparison between the Markov approximation and numerical simulations. Bull Seism Soc Am 90:914-928. 
Fúster JM (1975) Las Islas Canarias: un ejemplo de evolución temporal y espacial del volcanismo oceánico. Est Geol 31: 439-463.

Gusev A, Abubakirov IR (1999a) Vertical profile of effective turbidity reconstructed from broadening of incoherent body-wave pulses-I. General approach and the inversion procedure. Geophys J Int 136:295-308.

Gusev A, Abubakirov IR (1999b) Vertical profile of effective turbidity reconstructed from broadening of incoherent body-wave pulses-II. Application to Kamchatka data. Geophys J Int 136:309-323.

Hoernle K, Schmincke HU (1993) The role of partial melting in the 15-Ma geochemical evolution of Gran Canaria: a blob model for the Canary hotspot. J Petrol 34: 599-626.

Hoshiba M, Sato H, Fehler M (1991) Numerical basis of the separation of scattering and intrinsic absorption from full seismogram envelope: a Monte-Carlo simulation of multiple isotropic scattering. Pap Meteorol Geophys 42: 65-91.

Mézcua J, Buforn E, Udías A, Rueda J (19929 Seismotectonics of the Canary Islands. Tectonophysics 208: 447-452.

Morgan WJ (1971) Convection plumes in the lower mantle. Nature 230:42-43.

Nishigami K (1991) A new inversion method of coda waveforms to determine spatial distribution of coda scatterers in the crust and uppermost mantle. Geophys Res Lett 18:2225-2228.

Obara K, Sato H (1995) Regional differences of random inhomogeneities around the volcanic front in the Kanto-Tokai area, Japan, revealed from the broadening of S wave seismogram envelopes. J Geophys Res 100:2103-2121.

Petukhin AG, Gusev AA (2003) The duration-distance relationship and average envelope shapes of small Kamchatka earthquakes. Pure Appl Geophys 160:1717-1743.

Ranero CR, Torné M, Banda E (1995) Gravity and multichannel seismic reflection constraints on the lithospheric structure of the Canary swell. Mar Geophys Res 17: 519-534.

Roest WR, Dañobeitia JJ, Verhoef J, Collete BJ (1992) Magnetic anomalies in the Canary Basin and the Mesozoic evolution of the Central North Atlantic. Mar Geophys Res 14: 1-24. 
Saito T, Sato H, Ohtake M (2002) Envelope broadening of spherically outgoing waves in threedimensional random media having power law spectra. J Geophys Res 107. doi:10.1029/2001JB000264.

Saito T, Sato H, Ohtake M, Obara K (2005) Unified explanation of envelope broadening and maximum-amplitude decay of high-frequency seismograms based on the envelope simulation using the Markov approximation: forearc side of the volcanic front in northeastern Honshu, Japan. J Geophys Res 110, B01304. doi:10.1029/2004JB003225.

Sato H (1989) Broadening of seismogram envelopes in the randomly inhomogeneous lithosphere based on the parabolic approximation: southeastern Honshu, Japan. J Geophys Res 94:1773517747.

Sato H, Fehler M C (1998) Seismic wave propagation and scattering in the heterogeneous earth. AIP Series in Modern Acoustics and Signal Processing, Springer-Verlag, New York.

Socías I, Mézcua J (1996) Levantamiento aeromagnético del archipiélago canario. Instituto Geográfico Nacional, Madrid 35: 1-28.

Takahashi T, Sato H, Nishimura T, Obara K (2007) Strong inhomogeneity beneath Quaternary volcanoes revealed from the peak delay analysis of $S$-wave seismograms of microearthquakes in northeastern Japan. Geophys J Int 168:90-99. doi:10.1111/j.1365-246X.2006.03197.x.

Tripathi JN, Sato H, Yamamoto M (2010) Envelope broadening characteristics of crustal earthquakes in northeastern Honshu, Japan. Geophys J Int 182:988-1000. doi: 10.1111/j.1365246X.2010.04657.x.

Ugalde A, Carcolé E, Tripathi JN (2006) Spatial distribution of scatterers in the crust by inversion analysis of coda envelopes: a case study of Gauribidanur seismic array (southern India). Geophys J Int 166:782-794.

Ugalde A, Tripathi JN, Hoshiba M, Rastogi BK (2007) Intrinsic and scattering attenuation in western India from aftershocks of the 26 January, 2001 Kachchh earthquake. Tectonophysics 429:111-123.

Verhoef J, Collette BJ, Dañobeitia JJ, Roeser HA, Roest WR (1991) Magnetic anomalies off West-Africa $\left(20-38^{\circ} \mathrm{N}\right)$. Mar Geophys Res 13: 81-103.

Wu RS (1985) Multiple scattering and energy transfer of seismic waves: Separation of scattering effect from intrinsic attenuation, I, Theoretical modeling. Geophys J R Astron Soc 82:57-80. 


\section{Figure captions}

Fig. 1 Top: Topographic map of the Canary Islands region based on GMT ETOPO2v2 data [U.S. National Oceanic and Atmospheric Administration, http://www.ngdc.noaa.gov/mgg/fliers/06mgg01.html]. The contour interval shown is 500 meters. The location of the seismic stations is indicated by solid triangles. The epicenters (open circles) of the earthquakes available are also plotted. Grey lines are the seismic ray paths used in the analysis. Bottom: Projection of the focal depth of the hypocenters used along the longitude axis.

Fig. 2 Example of the velocity and bandpass-filtered recording for an actual seismogram (top) and its normalized rms envelope for the frequency band 4-8 Hz (bottom). The vertical grey dotted line represents the S-wave onset. The arrows show the measurement of $t_{p}$ and $t_{q}$

Fig. 3 Logarithmic plot of the S-wave peak delay times ( $t_{p}$, open circles) and envelope broadening parameters ( $t_{q}$, solid triangles) against hypocentral distance for the four frequency bands studied. The black dotted and grey solid lines represent the best linear fits in logarithmic scale of $t_{p}$ and $t_{q}$, respectively. Two hypocentral depth ranges are considered: $\quad 18$ km (left) and $18 \quad 80$ km (right).

Fig. 4 Plot of the regression slopes $B_{p}$, and $B_{q}$ as a function of frequency for the two hypocentral depth ranges considered: $\quad 18 \mathrm{~km}$ and $18 \quad 80 \mathrm{~km}$.Fig. 5 Path dependence of S-wave envelopes for earthquakes (1) and (2) in the Canary Islands region. The rms envelopes of 2-4 Hz bandpass-filtered seismograms observed at different stations are shown. The vertical grey dotted lines mark the S-wave onset, the solid and open triangles indicate the measurement of $t_{p}$ and $t_{q}$, respectively.

Fig. 6 Normalized $t_{p}$ (solid circles) and $t_{q}$ (white triangles) measurements as a function of frequency for stations CHIO, EBAJ, EOSO, CHIE, EGOM, and CFTV. The reference frequency band is 4-8 Hz. Small and large symbols indicate individual measurements and the average values, respectively. Fig. 7 Minimum value distribution of the logarithmic deviation of S-wave peak delay time, $\quad$ for the frequency bands $2-4 \mathrm{~Hz}, 4-8 \mathrm{~Hz}$, and 8-12 Hz. Slices at 0-11 km and 11$22 \mathrm{~km}$ in depth for upper mantle events (18 $80 \mathrm{~km})$ are shown. Blue and orange colors indicate negative and positive values of respectively.

Fig. 8 Comparative plot of the regression slopes of (equation 1) obtained in this study for upper mantle events $(h>18 \mathrm{~km})$ and in other regions. 


\section{Table captions}

Table 1 S-wave velocity model of the Canary Islands region.

$\begin{array}{llrl}\text { Table } 2 \text { Estimated regression coefficients and } \quad \text { of time lags } t_{p} \text { and } t_{q} \text { along with } \\ \text { their error estimates and correlation coefficients } & \text { of the fit for the two hypocentral depth }\end{array}$ ranges considered: $\quad 18 \mathrm{~km}$ and $18 \quad 80 \mathrm{~km}$.. 


\section{FIGURE 1}
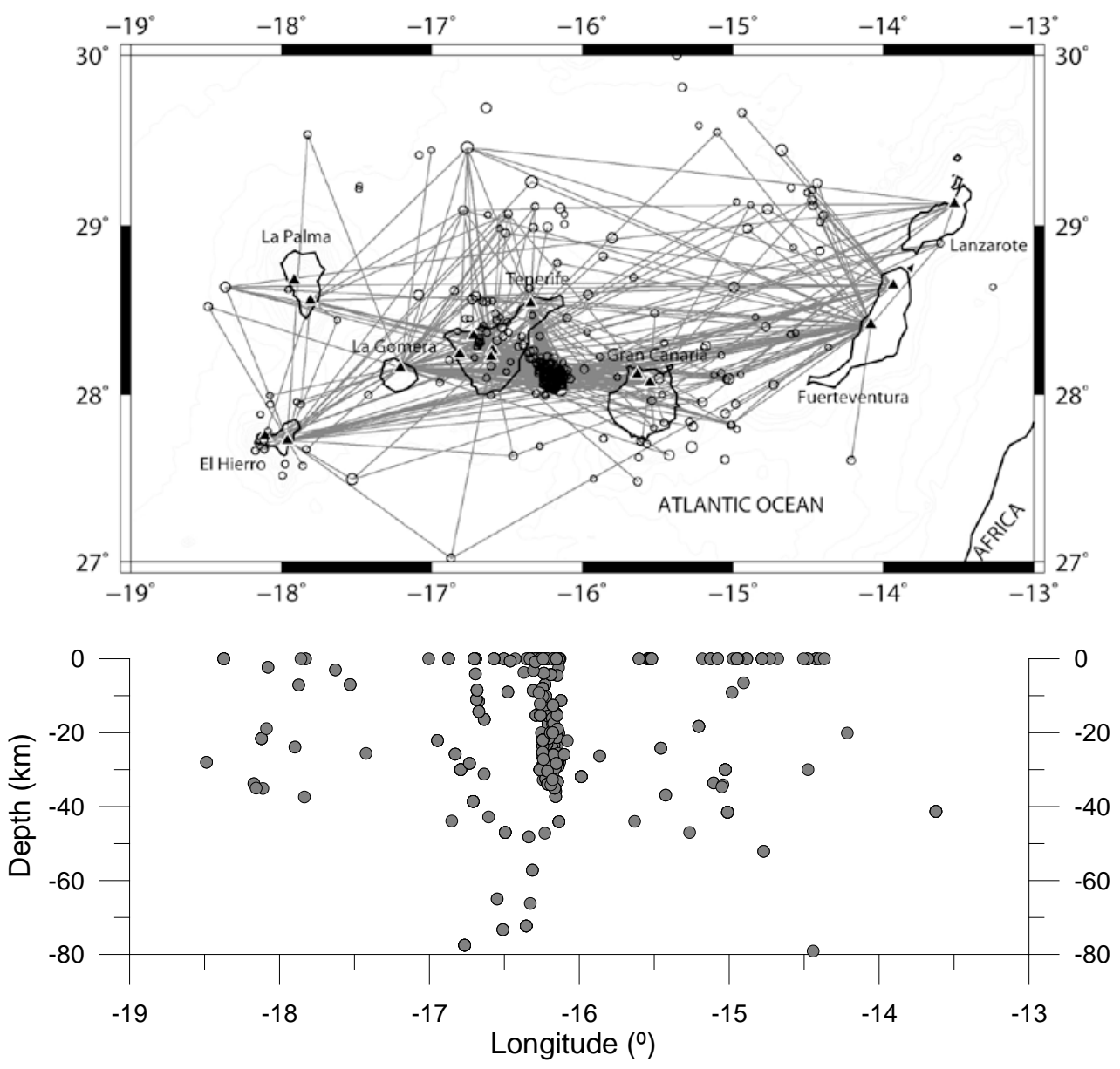
FIGURE 2

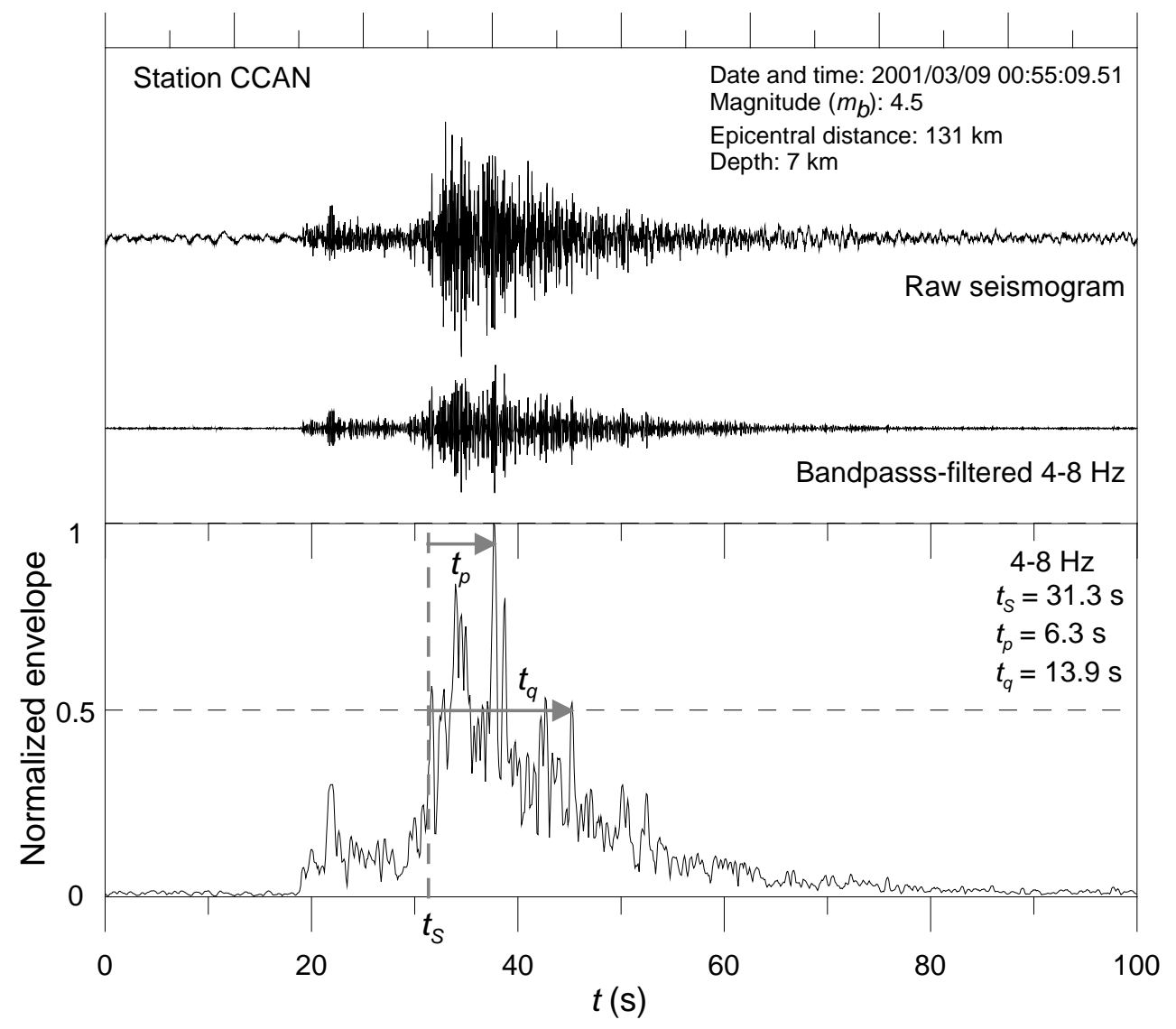




\section{FIGURE 3}

\section{$t_{p}(\mathbf{s}): \bigcirc t_{q}(\mathbf{s}): \triangle$}

\section{$h \leq 18$ km}

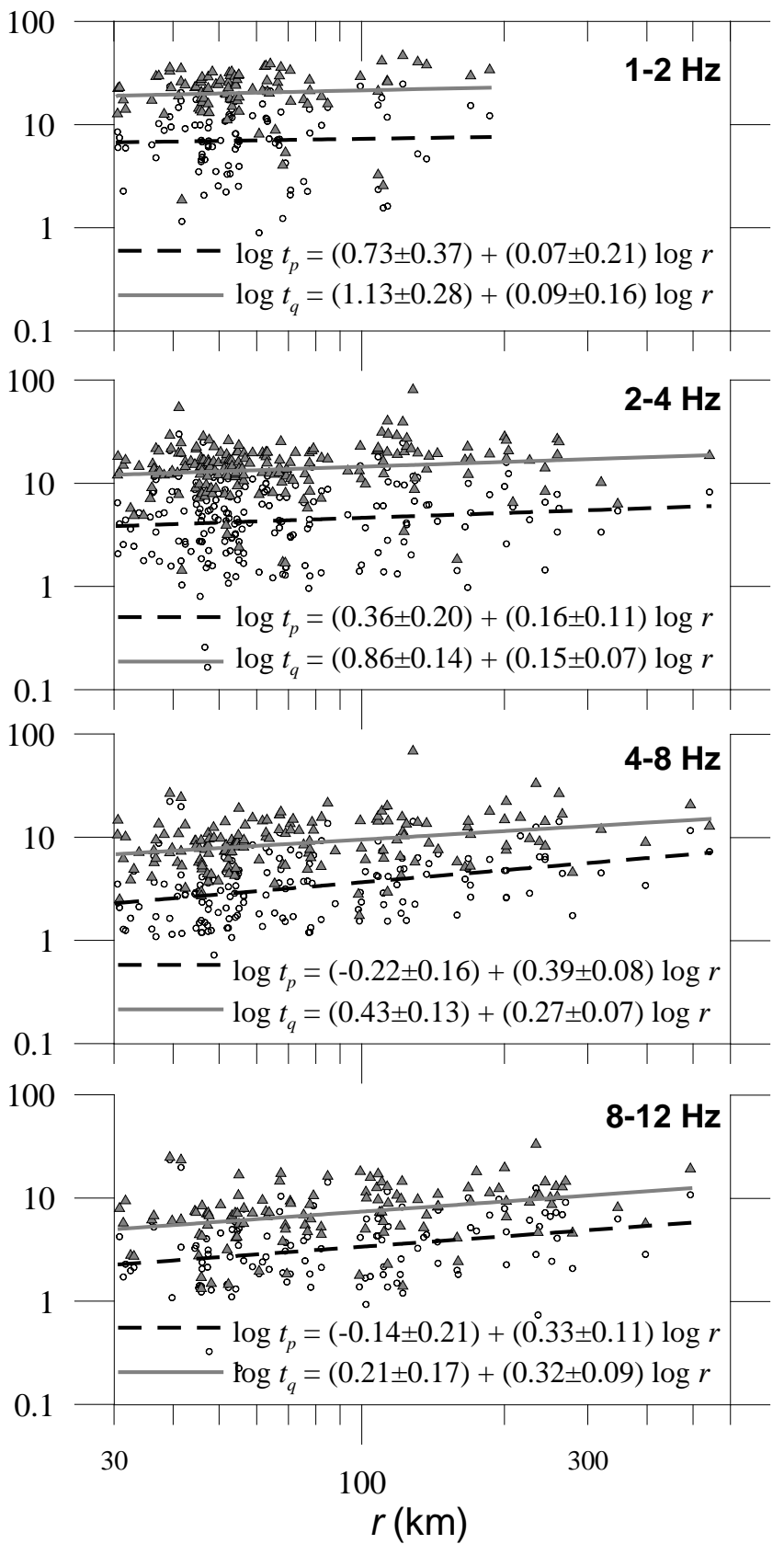

$h>18 \mathrm{~km}$
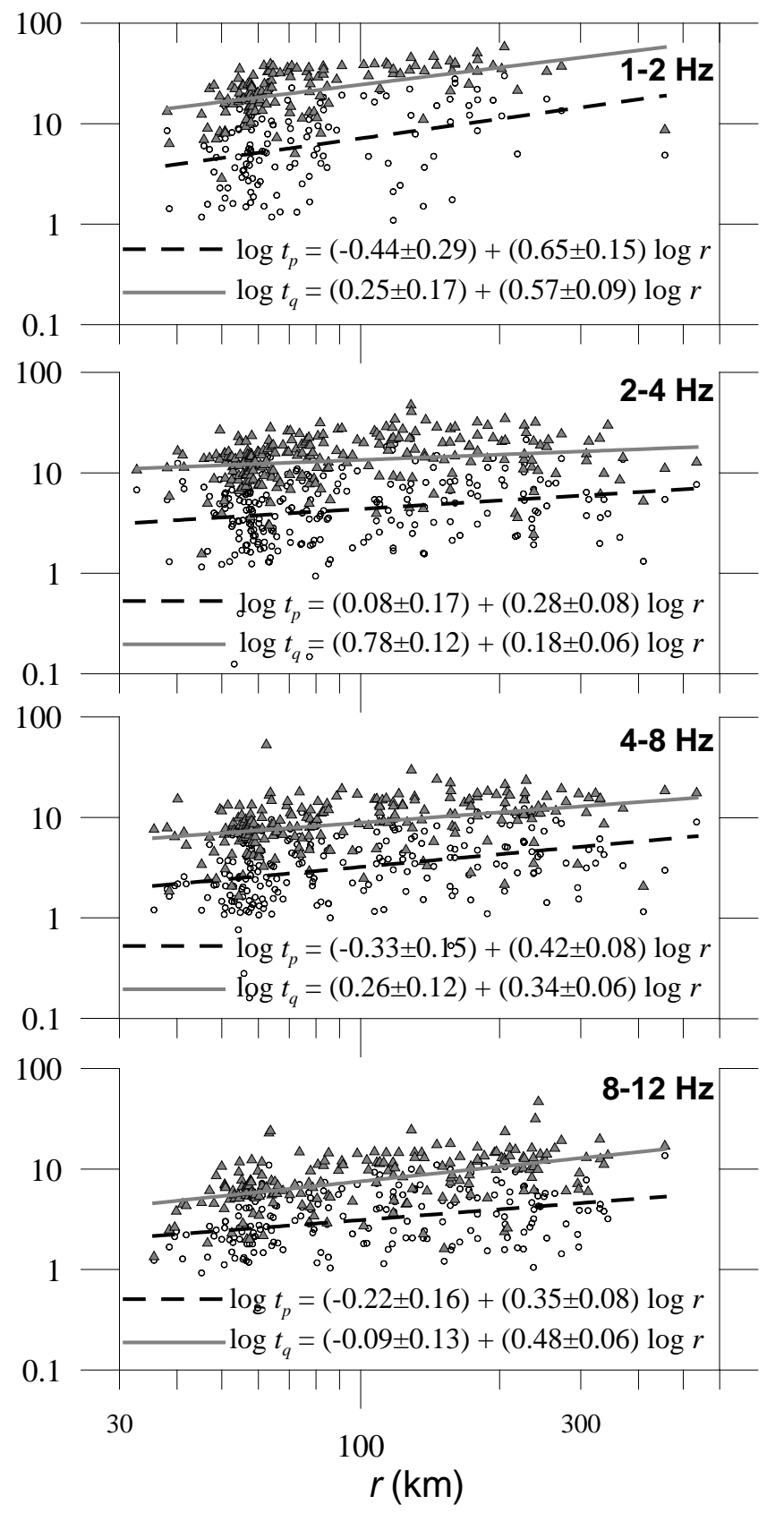


\section{FIGURE 4}
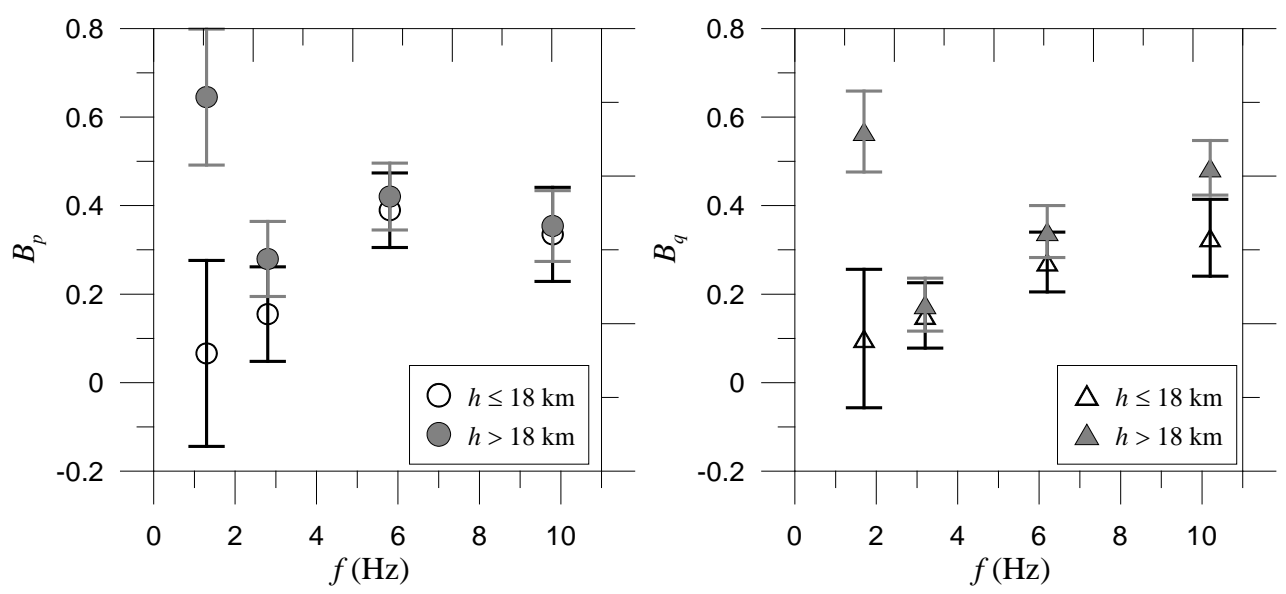
FIGURE 5

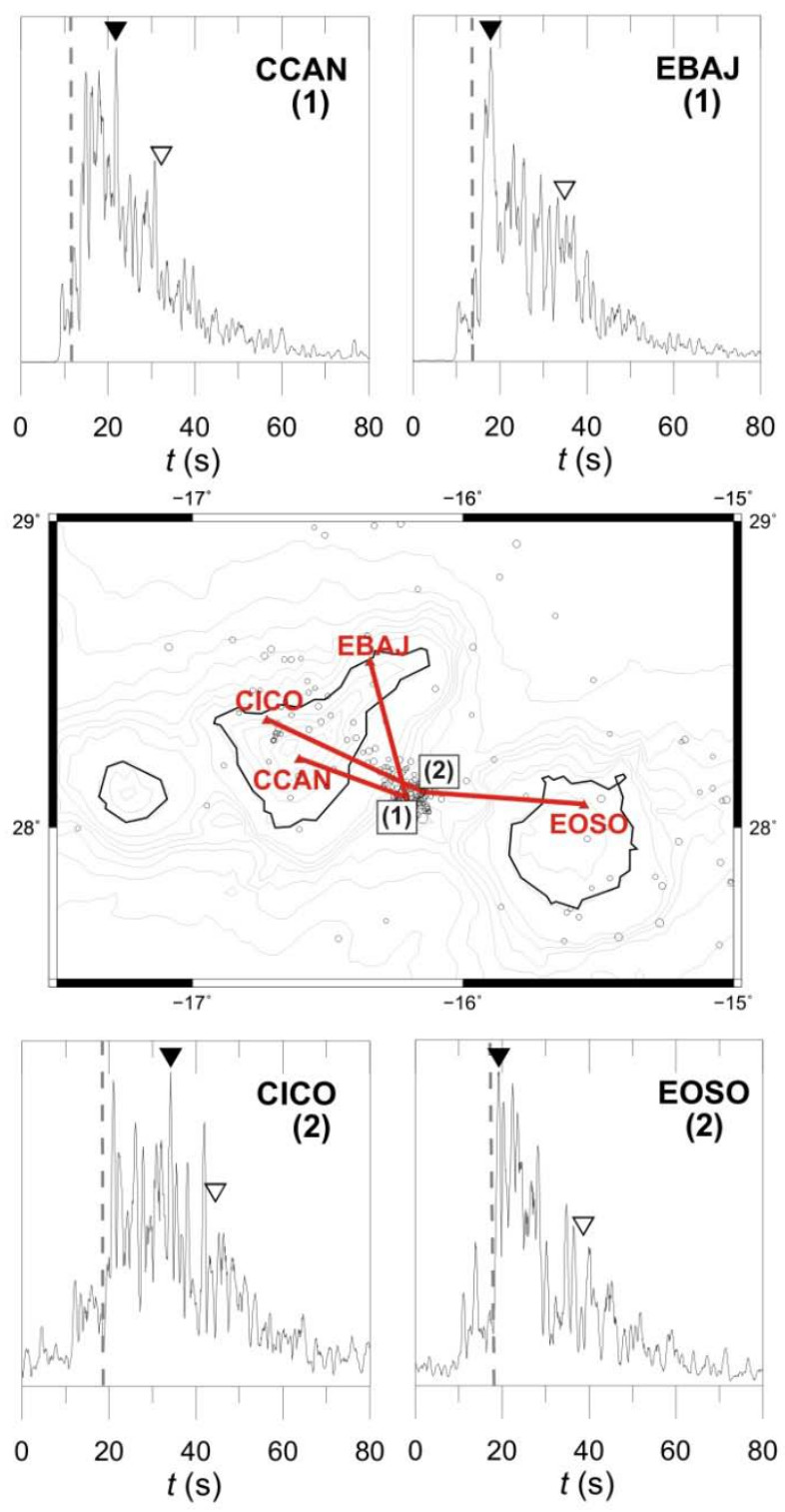




\section{FIGURE 6}

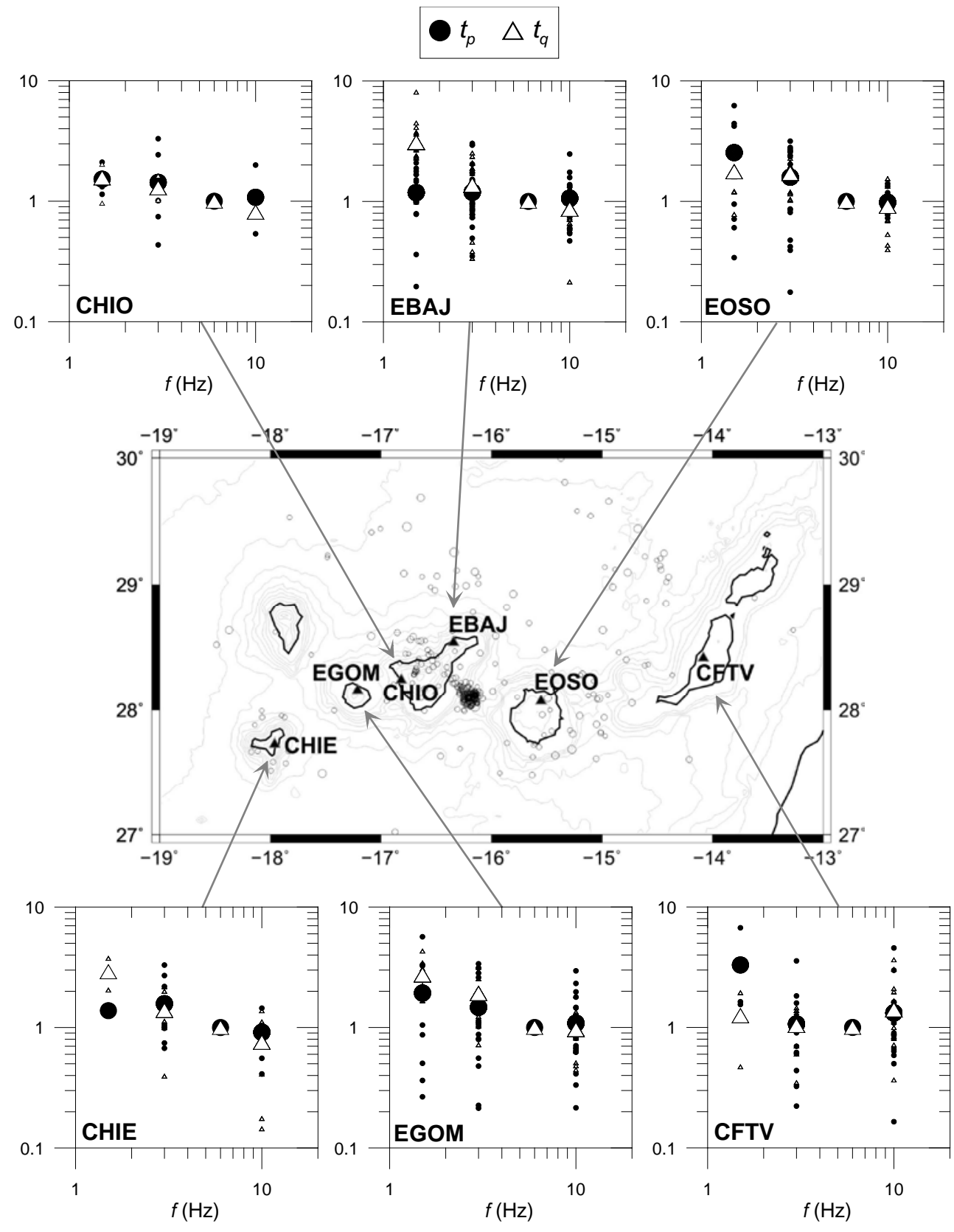




\section{FIGURE 7}
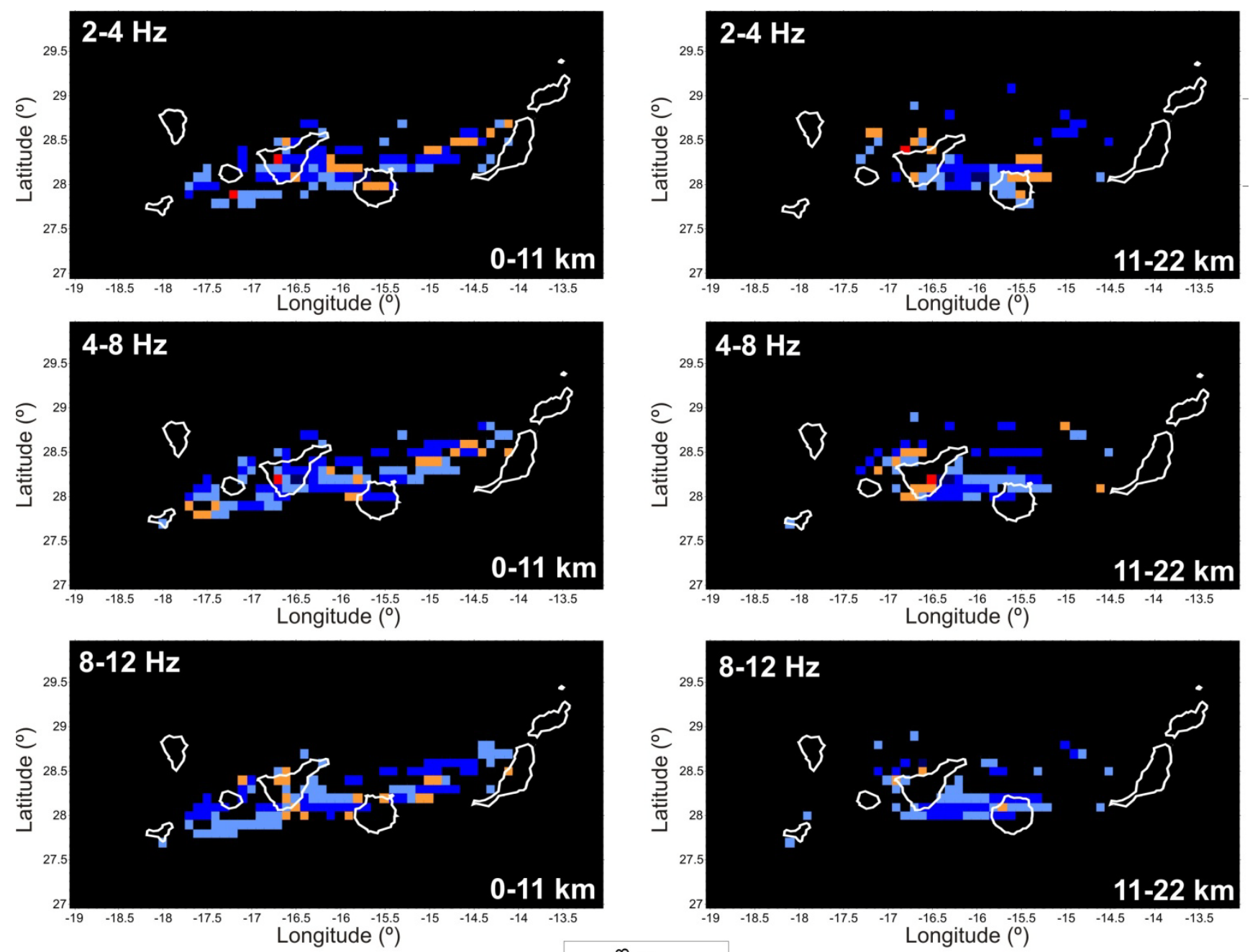

T:

웅ㅇำ

口а

$\Delta \log t_{p}$ 
FIGURE 8

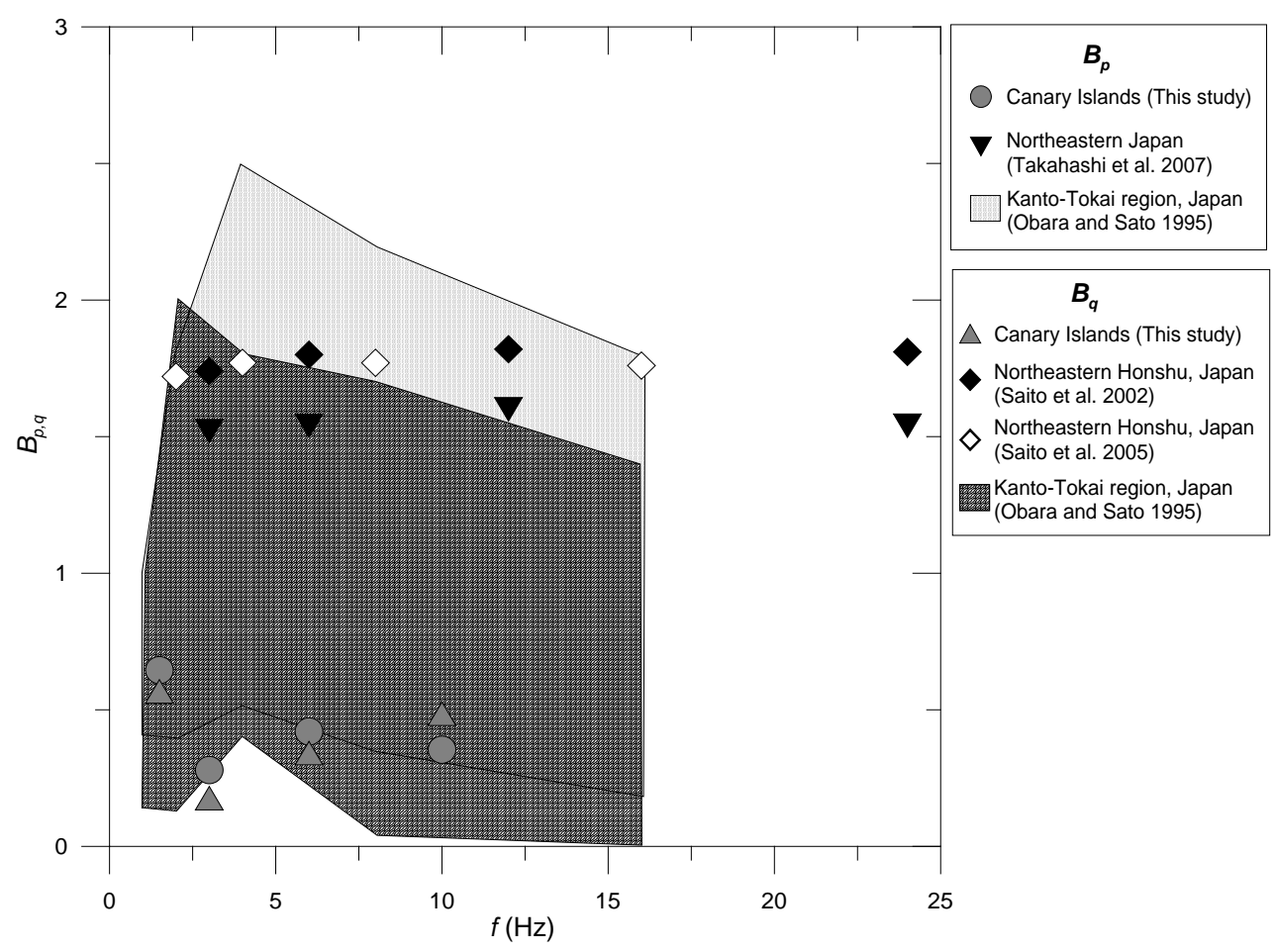

